\title{
Practicability of Submandibular Gland in Squamous Cell Carcinomas of Oral Cavity
}

\author{
Monika S. Malgonde $\cdot$ Manoj Kumar
}

Received: 17 October 2014/Accepted: 15 November 2014/Published online: 22 November 2014

(C) Association of Otolaryngologists of India 2014

\begin{abstract}
Current surgical treatment for oral cancers includes wide surgical excision of the primary lesion with appropriate neck dissection. Although tumor metastasis to the gland is uncommon, submandibular glands are frequently excised as a part of neck dissection because of their proximity to the primary lesion and afferent lymph nodes. Herein, we have retrospectively investigated the rate of involvement of submandibular glands in oral cavity tumors, and discussed the feasibility about resection of the submandibular glands. 94 patients undergoing 98 supraomohyoid neck dissections for oral cavity squamous cell carcinoma from 1st May 2010 till 31st May 2014 were retrospectively evaluated. Inclusion criteria were histopathologically confirmed squamous cell carcinoma of the oral cavity and surgery as the primary treatment modality, consisting of resection of the primary lesion with additional neck dissection, including at least one submandibular gland. Among the 98 specimens involvement of submandibular gland was seen only in 3 patients $(3.06 \%)$. Submandibular gland involvement was due to direct invasion of primary tumors and via the metastatic periglandular lymph nodes. Decision regarding excision of the gland should be done during the operation
\end{abstract}

M. S. Malgonde ( $\square)$

Department of ENT, Lilavati Hospital \& Research Centre,

Mumbai, Maharashtra, India

e-mail: dr.mona38@gmail.com

\section{Kumar}

Department of Orthopedics, Lilavati Hospital and Research

Centre, Mumbai, India

e-mail: manoj@mgims.ac.in;

manoj_mgims0234@rediffmail.com with the help of visual examination and frozen sections instead of preoperative planned resection. Preserving submandibular gland is both technically as well as oncologically feasible and can achieve potential benefit in preserving the submandibular salivary flow.

Keywords Oral cavity squamous cell carcinoma . Neck dissection · Preservation · Submandibular gland

\section{Introduction}

In high risk countries such as Sri Lanka, India, Pakistan and Bangladesh, oral cancer is the most common cancer in men and may account for up to $30 \%$ of all new cases of cancer compared to $2 \%$ in the UK and $8 \%$ in France [1]. Current surgical treatment includes wide surgical excision of the primary lesion with appropriate neck dissection. Neck metastases are most frequently observed in levels I-III, but rarely in level IV [2]. The submandibular glands are located in level-Ib where rich lymphatic tissues surround them. A selective lymphadenectomy clearing nodal levels I, II and III (so-called supraomohyoid neck dissection, or SOND) is traditionally recommended for N0 patients with primary squamous cell cancers of oral cavity. While some have also advocated the use of SOND in N1 necks without evidence of extra capsular spread [3]. Although tumor metastasis to the gland is uncommon, submandibular glands are frequently excised as a part of neck dissection because of their proximity to the primary lesion and afferent lymph nodes. Herein, we have retrospectively investigated the rate of involvement of submandibular glands in oral cavity tumors, and discussed the feasibility about resection of the submandibular glands. 


\section{Materials and Methods}

94 patients undergoing 98 supraomohyoid neck dissections for oral cavity squamous cell carcinoma from 1st May 2010 till 31st May 2014 were retrospectively evaluated. Inclusion criteria were histopathologically confirmed squamous cell carcinoma of the oral cavity and surgery as the primary treatment modality, consisting of resection of the primary lesion with additional neck dissection, including at least one submandibular gland. Patients with tumor histology other than squamous cell carcinoma or with a history of previous head and neck radiotherapy were excluded. The postoperative histopathological reports were evaluated to see for the involvement of submandibular gland.

\section{Results}

The clinical charts of 94 patients were reviewed, 66 were male $(70.21 \%)$ and 28 female $(29.78 \%)$ with a mean age of 51 years (range: $35-76$ ). The demographic and clinical characteristics are presented in Table 1.

Surgery in 4 of the patients $(4.25 \%)$ involved bilateral neck dissection with bilateral submandibular gland excision, while in the remaining 90 patients $(95.74 \%)$ onesided neck dissection with unilateral submandibular gland excision was performed. Consequently, 94 patients and 98 submandibular glands were included in the study. Table 2 shows the distribution of primary tumor sites.

Table 1 Showing demographic and clinical characteristics of the patients

\begin{tabular}{ll}
\hline Case distribution & \\
\hline No. of patients & 94 \\
M:F & $2.3: 1$ \\
Age range & $35-76$ \\
Mean (years) & 51 years \\
\hline
\end{tabular}

Tumor involvement of the submandibular gland was observed in 3 patients. Of these, 2 were due to direct invasion of the primary lesion (1 tongue tumors with T4N2b lesion, 1 FOM tumors with T4N2b lesion). One of the gland displayed invasion through the metastatic periglandular lymph node (buccal mucosa tumor with a T4N2b lesion). In 35 early staged patients (stage $1 \& 2$ ) none had any involvement of the submandibular gland whereas 3 of the patients in advanced stages (stage $3 \& 4$ ), of the 59 patients, had submandibular gland involvement $(5.08 \%)$. Submandibular gland involvement was mostly due to the invasion of primary tumors due to their proximity.

\section{Discussion}

Major product of submandibular salivary gland is saliva. Submandibular gland secretes most of the unstimulated, resting saliva and are responsible for most of the protective functions of saliva. Mucus cells secrete mucus which aids in lubrication of food and serous cells produce salivary amylase which aids in breakdown of starches. The presence of bicarbonate in saliva makes it slightly alkaline, which is important in buffering acidic bacterial enzymes, and it plays an important role in tooth integrity by helping mineralization. Its antimicrobial activity comes from IgA, lactoferrin and other enzymes vital to the maintenance of healthy hard (teeth) and soft (mucosa) oral tissues. Severe reduction of salivary output not only results in a rapid deterioration in oral health but also has a detrimental impact on the quality of life for the sufferer. Patients suffering from dry mouth can experience difficulty with eating, swallowing, speech, the wearing of dentures, trauma to and ulceration of the oral mucosa, taste alteration, poor oral hygiene, a burning sensation of the mucosa, oral infections including Candida and rapidly progressing dental caries.

Often an adjuvant treatment like radiotherapy damages the salivary glands and aggravates xerostomia. Swallowing is also compromised by the xerostomia due to salivary gland dysfunction arising from the direct effects of radiotherapy on these tissues. One of the important complications of

Table 2 Site wise distribution of tumors

\begin{tabular}{|c|c|c|c|c|c|}
\hline Oral cavity subsite & No. $(\%)$ & Stage I & Stage II & Stage III & Stage IV \\
\hline Lip & $2(2.12 \%)$ & - & 2 & - & - \\
\hline Tongue & $34(36.17 \%)$ & - & 8 & 25 & 1 \\
\hline FOM & $12(12.76 \%)$ & 1 & 4 & 6 & 1 \\
\hline Buccal mucosa & $46(48.93 \%)$ & 4 & 16 & 23 & 3 \\
\hline Hard palate & - & - & - & - & - \\
\hline \multirow[t]{2}{*}{ RMT } & - & - & - & - & - \\
\hline & & $5(5.3 \%)$ & $30(31.9 \%)$ & $54(57.4 \%)$ & $5(5.3 \%)$ \\
\hline
\end{tabular}


xerostomia is osteoradionecrosis due to plaques, gingivitis and periodontitis in the absence of salivary protection. Mucous glands are less sensitive. Many changes in salivary glands at $60 \mathrm{~Gy}$ are reversible, but beyond this, permanent damage occurs [4]. RT decreases salivary volume and $\mathrm{pH}$, and changes its composition [5]. When the submandibular glands are excised, these processes occur more rapidly and patients may cease RT. The use of the three-dimensional planning technique (3-DTP) and conformational dose delivery techniques have been shown to reduce the radiation damage to the parotid and other salivary glands and thus to decrease post irradiation xerostomia [6,7]. With the use of intensity-modulated radiation therapy (IMRT), the frequency of xerostomia decreases and preservation of the submandibular glands during surgery becomes more important.

According to Rouviere, there are 5 lymph node groups in this region: preglandular, prevascular, retrovascular, retroglandular and intracapsular [2]. DiNardo added the "deep submandibular node" to this group [8]. Among these, the perivascular nodes (comprising prevascular and retrovascular nodes) are the most important because they are the primary afferent draining nodes of the oral cavity. Perivascular node involvement in FOM and tongue cancers is seen in about 5-7\% of cases [9]. Technically, the lymphatic compartment of level IB, between the superficial and deep layers of the cervical fascia, can be easily separated from the parenchyma of the submandibular gland once the submandibular fascia is incised. In suitable cases, it is technically feasible to remove all the lymph nodes in sublevel IB and preserve the submandibular gland [10]. Metastasis to the submandibular gland itself is extremely rare and if removal of the lymph nodes of sublevel IB is imperative, it seems feasible to preserve the submandibular gland, unless it is involved by direct tumor extension of the primary tumor or the adjacent metastatic lymph nodes [11]. Basaran et al. also suggested that it is appropriate to preserve the gland unless the primary tumor or metastatic regional lymphadenopathy is adherent to the gland [12].

Some surgeons feel that preserving submandibular gland can cause persistent palpable mass in the site of previous lymphadenectomy and this preserved gland often scars and causes concern for both patient and otolaryngologist-head neck surgeon who follows the patient.

Among the 98 specimens involvement of submandibular gland was seen only in 3 patients $(3.06 \%)$ ). Over the years there has been a trend towards more conservative approaches to neck dissection like functional neck dissection. Decision regarding excision of the gland should be done during the operation with the help of visual examination and frozen sections instead of preoperative planned resection. We conclude that preserving submandibular gland is both technically as well as oncologically feasible and can achieve potential benefit in preserving the submandibular salivary flow. Oncologically preservation submandibular glands in cases where selective neck dissections are indicated is safe and sound.

Conflict of Interest None.

\section{References}

1. Sankaranarayanan R, Masuyer E, Swaminathan R, Ferlay J, Whelan S (1998) Head and neck cancer: a global perspective on epidemiology and prognosis. Anticancer Res 18:4779-4786

2. Rouviere H, Tobies MJ (1938) Trans: Anatomy of the human lymphatic system. Edwards Brothers, Ann Arbor, pp 86-88

3. Byers RM, Weber R, Andrews T et al (1997) Frequency and therapeutic implications of "skip metastasis" in the neck from squamous carcinoma of the oral tongue. Head Neck 19:14-19

4. Reddy SP, Leman CR, Marks JE et al (2001) Parotid-sparing irradiation for cancer of the oral cavity: maintenance of oral nutrition and body weight by preserving parotid function. Am J Clin Oncol 24:341-346

5. Chambers MS, Garden AS, Kies MS et al (2004) Radiationinduced xerostomia in patients with head and neck cancer: pathogenesis, impact on quality of life, and management. Head Neck 26:796-807

6. Henson BS, Inglehart MR, Eisbruch A, Ship JA (2001) Preserved salivary output and xerostomia-related quality of life in head and neck cancer patients receiving parotid -sparing radiotherapy. Oral Oncol 37:84-93

7. Malouf JG, Aragon C, Henson BS, Eisbruch A, Ship JA (2003) Influence of parotid-sparing radiotherapy on xerostomia in head and neck cancer. Cancer Detect Prev 27:305-310

8. DiNardo LJ (1998) Lymphatics of the submandibular space: an anatomic, clinical, and pathologic study with applications to floor-of-mouth carcinoma. Laryngoscope 108:206-214

9. Lim YC, Kim JW, Koh YW et al (2004) Perivascular-submandibular lymph node metastasis in squamous cell carcinoma of the tongue and floor of mouth. Eur J Surg Oncol 30:692-698

10. Dhiwakar M, Ronen O, Malone J et al (2011) Feasibility of submandibular gland preservation in neck dissection: a prospective anatomic-pathologic study. Head Neck 33:603-609

11. Takes RP, Robbins KT, Wooglar JA et al (2011) Questionable necessity to remove the submandibular gland in neck dissection. Head Neck 33:743-745

12. Basaran B, Ulusan M, Orhan KS, Gunes S, Suoglu Y (2013) Is it necessary to remove submandibular glands in squamous cell carcinomas of the oral cavity? Acta Otorhinolaryngol Ital 33(2):88-92 